\title{
Centinelas: un proyecto de integración y acción ciudadana
}

\author{
Verónica L. Romero', Mariana Raño², M. Belén Natalini'3 ${ }^{3}$ M. Angelina \\ Godoy $^{4}$, R. Florencia Quijano ${ }^{4}$, Martín Sánchez ${ }^{4}$, Rodrigo Bay Joulia ${ }^{5}$, \\ Daniela Pucheta5 ${ }^{5}$ Débora Gilles5 ${ }^{5}$ Bárbara G. Romero ${ }^{6}$, Rocío A. Alegre5, \\ Joaquín Mayer5, Mauricio Landi5, Martín M. Kowalewski7 (*)
}

\section{Resumen}

Este proyecto es una tarea de extensión desde el ámbito de la investigación de monos aulladores negros y dorados (Alouatta caraya) y de zorros (Cerdocyon thous, Lycalopex gymnocercus) que habitan en las zonas de interfase urbano-rural de la Estación Biológica Corrientes (EBCo, CCT, CONICET) y un proyecto de UNNE+SALUD 2019. Los objetivos generales incluyeron proponer acciones para la detección temprana de ciertas enfermedades en la comunidad desde la participación ciudadana. Se efectuaron una serie de encuentros con ciudadanos/as de las localidades de la ciudad de Corrientes y Mburucuyá sobre el conocimiento de la ecología y epidemiología de estas especies centinelas. La meta es impulsar una red integrada por ciudadanos/as locales motivados/as por la búsqueda personal y colectiva de soluciones a problemáticas ambientales y epidemiológicas.

\footnotetext{
${ }_{1}^{1}$ Doctora en Biología, directora de Proyecto UNNE+SALUD (*), res. 0197/19 C. D., lorenvromer@gmail.com (*) 2Doctora en Biología, coordinadora de Proyecto UNNE+SALUD, res. 0197/19 C. D., marianarano@gmail.com ${ }^{3}$ Veterinaria, becaria doctoral EBCo, CCT, CONICET, belennatalini@gmail.com ${ }^{4}$ Guardaparques universitarios e integrantes de Proyecto UNNE+SALUD, FaCENA, UNNE.

${ }^{5}$ Estudiantes universitarios de la Facultad de Ciencias Exactas y Naturales y Agrimensura, integrantes de Proyecto de Extensión UNNE.

${ }^{6}$ Guia intérprete ambiental de la EBCo, integrante de Proyecto UNNE+SALUD, FaCENA, UNNE, barbaragraciela@hotmail.com.ar 'Doctor en Antropología, Director de EBCo, CCT, CONICET, martinkow@gmail.com

(*) Facultad de Ciencias Exactas y Naturales y Agrimensura, Avenida Libertad 5470, CP: 3400, ciudad de Corrientes labordon85,esteporcel,rocioayalazara@gmail.com
} 
En total se llevaron a cabo tres presentaciones del proyecto a la que asistieron un total de 120 personas, y ocho talleres con la participación de 62 personas entre ambas localidades, que consistieron en salidas de campo en áreas protegidas y rurales, práctica de análisis coproparasitológicos y elaboración de proyectos de acción. La red de "Centinelas en acción" en la región noreste de Argentina actuará como nodo de comunicación entre los profesionales, los participantes capacitados y los actores de zonas urbanas, periurbanas o rurales que coexistan y se relacionen con estas especies centinelas. Es de interés promover la sensibilización de la comunidad sobre estas temáticas de Salud pública y de Salud de los Ecosistemas, desde una actividad investigativa y de ciencia ciudadana, generando concientización y la implementación de acciones para asegurar la conservación de estas especies a largo plazo.

Palabras clave: enfermedades zoonóticas, salud del ecosistema, ciencia ciudadana, parabiólogos/as.

\section{Introducción}

La expansión de la población humana, y el crecimiento urbano, producen un cambio en la composición de los ambientes naturales; resultando en un aumento en la dinámica multidireccional de los patógenos (Gillespie et al. 2008, Bonilla-Aldana et al. 2020, Schemeller et al. 2020). Los países de Sudamérica son afectados con la destrucción del ambiente, y al mismo tiempo, se enfrentan hoy al uso egoísta, desmedido y perjudicial de los paisajes naturales por los países más desarrollados, corporaciones globales, y/o empresas vinculadas al agronegocio (Rodríguez Morales et al. 2011). En este sentido, la cadena casual entre el cambio global en el ambiente y los impactos sobre la salud puede ser bastante compleja y presentar efectos retardados (Navarro Gómez \& Ruiz Salgado 2016). Actualmente, estos cambios rápidos proponen un gran desafío en la conservación de la biodiversidad y la salud global (Kowalewski et al. 2011). Estas transformaciones, en gran medida, han sido responsables de la extinción de numerosas especies mediante la pérdida, fragmentación, deterioro o polución de sus hábitat (Navarro Gómez \& Ruiz Salgado 2016). En este contexto, es fundamental promover acciones de concientización, prevención y capacitación sobre enfermedades infecciosas emergentes y reemergentes. Cerca del 70\% de la enfermedades emergentes son de origen zoonótico, siendo estas enfermedades 
nuevas o bien presentes pero con extensión de su rango de distribución (Woolhouse et al. 2005, Jones et al. 2008).

Y en este contexto, algunos animales, por sus características comportamentales, de distribución y susceptibilidad a diversos patógenos pueden ser considerados como centinelas de la salud (Halliday et al. 2007). Una especie centinela es aquella que se expone a patógenos/contaminantes en el ambiente y es estudiada regularmente para identificar agentes (o sus efectos) que sean potencialmente nocivos para la salud poblacional de otros animales de la misma especie o especies diferentes (Kowalewski \& Gillespie 2018). Los atributos que definen a estas especies centinela son la respuesta medible a la presencia de un patógeno, la interacción tanto con el patógeno como con la población objetivo y deben presentar una densidad en el ambiente que permita su identificación y posible captura (Halliday et al., 2007). Así, por ejemplo, en el noreste argentino, los monos aulladores negros y dorados (Alouatta caraya) y zorros de mediano porte (Cerdocyon thous, Lycalopex gymnocercus) pueden ser considerados centinelas de algunas enfermedades infecciosas (Kowalewski et al. 2011, Kowalewski \& Gillespie 2018, Natalini 2018, Natalini et al. 2019). Siendo, la detección temprana de patógenos en estos animales centinelas relevantes para la salud humana y de otros animales silvestres, y las intervenciones e iniciativas de políticas públicas de salud (Reif 2011).

Este proyecto se encuentra a la vez enmarcado en los planteamientos de «Una sola salud», entendiendo la interrelación intima entre personas, animales y ecosistemas como una herramienta fundamental para cualquier trabajo de investigación, prevención y/o control de una enfermedad zoonótica. En este sentido, y en el marco de proyectos de investigación, integrantes de la Estación Biológica Corrientes (EBCo, CCT, CONICET), del Grupo de Estudios de Mamíferos (GEM) y de la Facultad de Ciencias Exactas y Naturales y Agrimensura (FaCENA, UNNE) hemos elaborado un proyecto dentro de UNNE+SALUD entre el 23 de mayo al 10 de diciembre de 2019. El mismo tuvo como meta el establecimiento de una red dentro de la provincia y la primera en Argentina, donde los/as participantes (parabiólogos/as) actúen como nodos de comunicación entre los profesionales y los actores locales en espacios de la comunidad como clubes de ciencias, escuelas secundarias, centros de salud o sectores de zonas urbanas, periurbanas o rurales que potencialmente coexistan 
con las especies centinelas. Un antecedente previo en Sudamérica que ha servido como base de este proyecto, fue la alianza entre un pueblo originario y un grupo conservacionista del Gran Chaco Boliviano para el relevamiento de mamíferos y el entrenamiento de parabiologos/as acciones que tenían por objetivo asegurar el sentimiento de apropiación en el proceso de conservación (Cuellar \& Noss 2014). Por lo tanto, en este proyecto, consideramos que los/las parabiólogos/as son personas con experticia en temas relacionados a mecanismos de transmisión de enfermedades zoonóticas y otras de interés sanitario regional que involucre mamíferos centinelas y promuevan la importancia de su rol en la prevención de epidemias y/o endemias junto a la conservación de ambientes saludables en la provincia de Corrientes.

\section{Metodología}

Este proyecto de UNNE+SALUD tiene como base la experiencia de investigaciones y otros proyectos de extensión sobre especies de mamíferos que actúan como centinelas de enfermedades zoonóticas y de los ambientes que habitan en la provincia de Corrientes. En total se efectuaron 3 encuentros y 8 talleres de capacitación en la FaCENA, la Facultad de Ciencias Veterinarias de la ciudad de Corrientes, y en el Instituto Superior de Formación Docente Mburucuyá (ISFD) de la localidad de Mburucuyá. Inicialmente, se realizó una charla de presentación de los fundamentos, objetivos y metodologías del proyecto, y se llevó a cabo una encuesta para conocer el perfil de los asistentes e inscripción de los interesados. En este aspecto, consideramos que una de las claves para una asociación exitosa entre los/as futuros/as parabiólogos/as con el equipo de trabajo fue evaluar los intereses de las partes interesadas, especialmente aquellos que se superponen, para comenzar con una base sólida de trabajo (Duboisset-Broust 2013). En total los/las destinatarios/as seleccionados/ as fueron 30 personas en la ciudad de Corrientes, entre ellos/as estudiantes de FaCENA, Veterinaria, naturalistas y guías de turismo, y 32 personas en Mburucuyá entre ellos/as estudiantes del Profesorado en Educación Secundaria en Biología (ISFD Mburucuyá), naturalistas y docentes.

Posteriormente, en cada localidad se realizaron cuatro talleres de contenido similar que involucraron actividades de campo (Fig.1) para el aprendizaje del reconocimiento de especies centinelas - mono aullador negro y dorado (Alouatta 
Fig. 1: Primer taller con actividades de campo en el Parque Provincial San Cayetano y EBCo (San Cayetano) y en Mburucuyá (Mburucuyá), durante junio 2019.

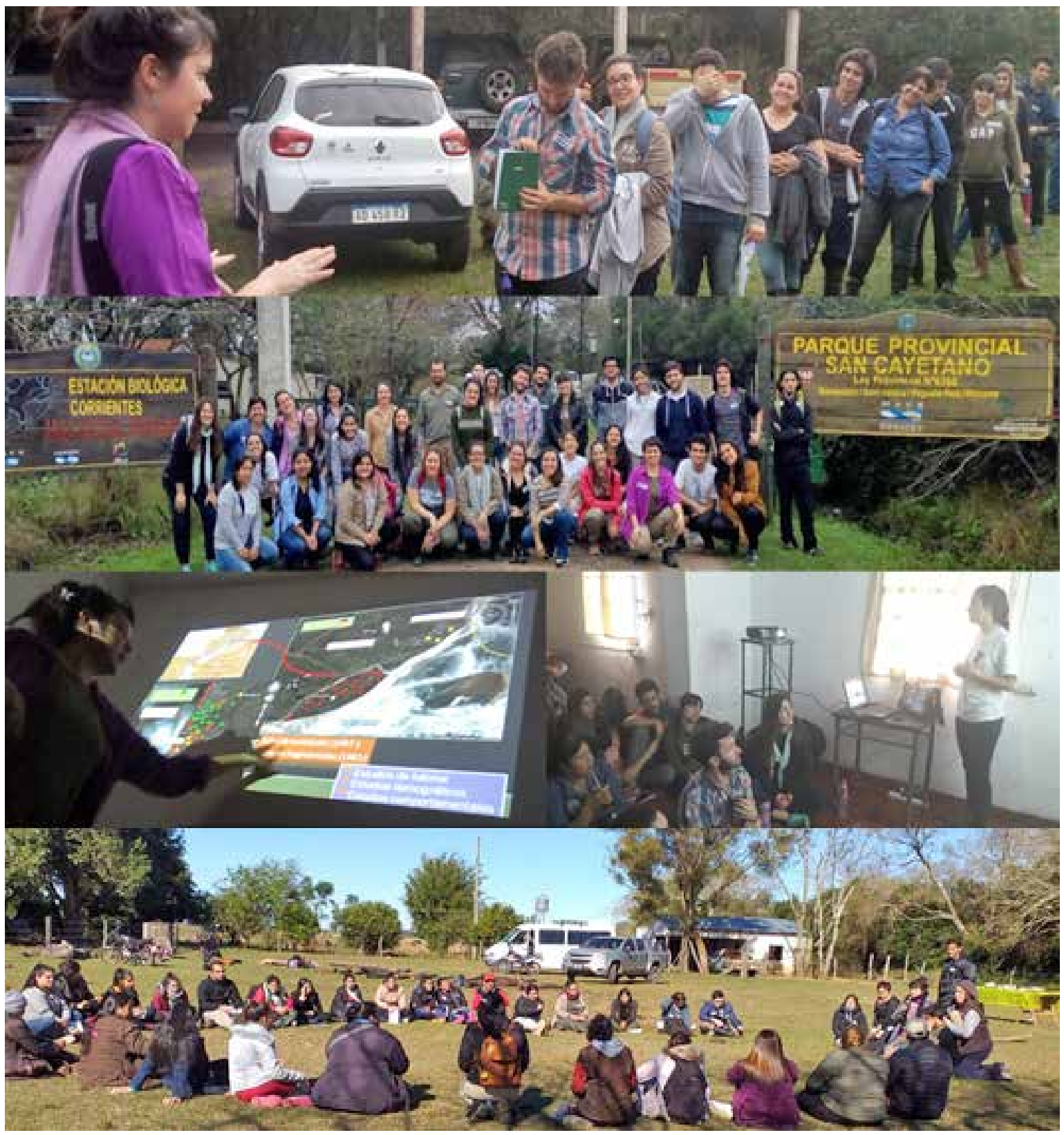

caraya) y zorros de mediano porte (Cerdocyon thous y Lycalopex gymnocercus) - en ambientes naturales y rurales - Parque Provincial San Cayetano y campo aledaño a Mburucuyá- junto a la forma de estudio de aspectos biológicos, ecológicos y epidemiológicos. Además, en este taller se enfatizó la importancia del contacto de los participantes con ambientes abiertos y biodiversos que resultan de beneficio positivo para la salud y el bienestar de las personas (Navarro Gómez \& Ruiz Salgado 2016). Luego, el segundo taller consistió en un trabajo de laboratorio para el 
aprendizaje y entrenamiento de técnicas rápidas de análisis coproparasitológicos (Fig. 2). Y, un tercer taller que consistió en reflexionar sobre el rol de los parabiólogos y un plan estratégico para el establecimiento de la Red Centinelas en acción (Fig. 3). La última etapa consistió en un cuarto taller donde los participantes expusieron distintas estrategias de acción con las especies centinelas y problemáticas locales que afectan la salud ambiental, y luego una etapa de ejecución de las mismas y una evaluación final.

En el cuarto taller, los participantes expusieron sus 12 proyectos con una presentación de 20 a 30 minutos, donde manifestaron fundamentos, objetivos, estrategias y plazos de las acciones. $\mathrm{Y}$ finalmente, se evaluaron estos proyectos por el equipo de trabajo para la continuidad de los mismos en una segunda etapa de UNNE+SALUD.

Fig. 2: Segundo taller sobre prácticas de análisis coproparasitológicos, en FaCENA (UNNE) y en el ISFD Mburucuyá (Mburucuyá) durante julio y agosto 2019.

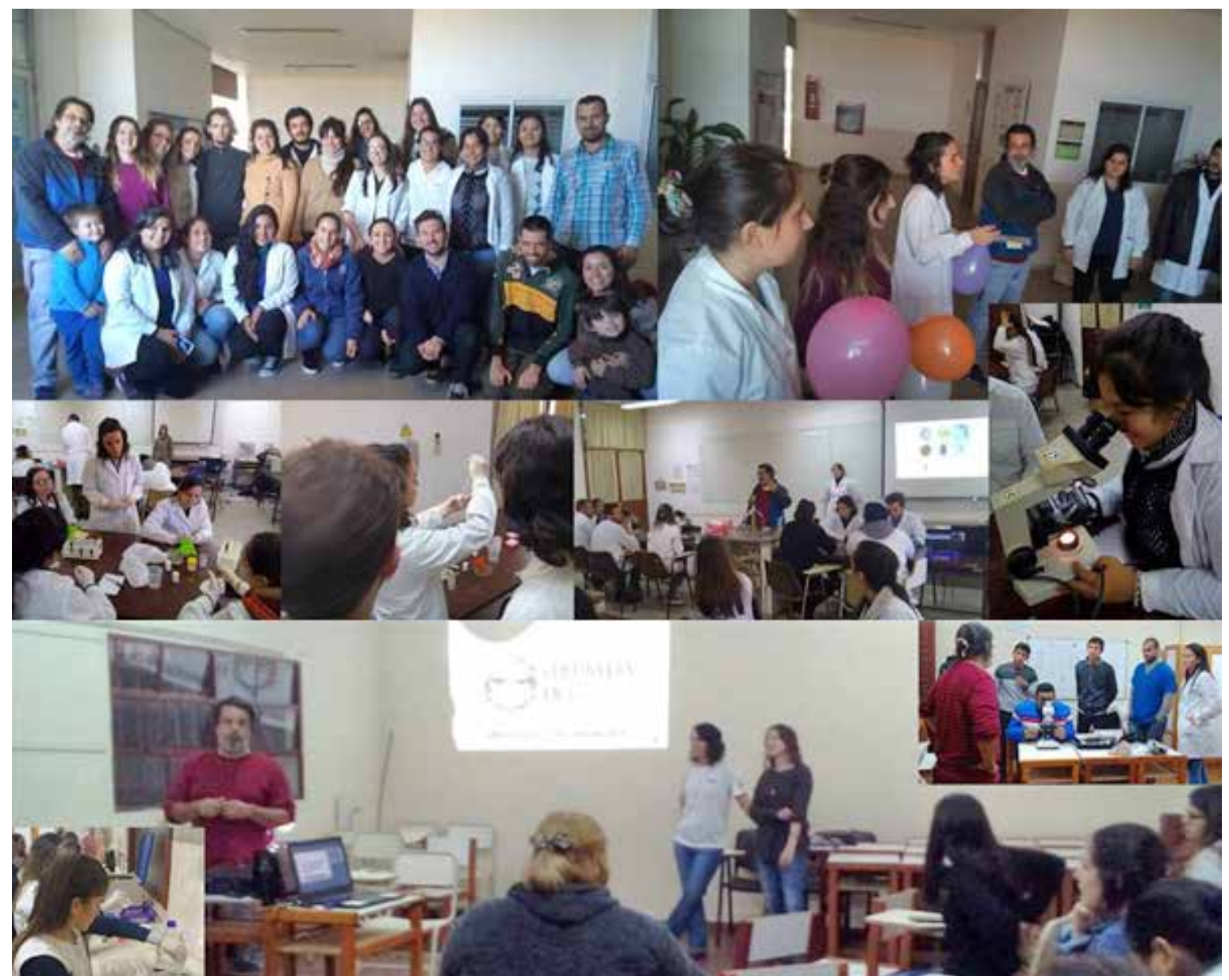


Fig. 3: Tercer taller sobre el rol de los parabiólógos/as y plan estratégico para el establecimiento de la Red en Corrientes durante septiembre 2019 en la FaCENA (Corrientes) y el ISFD Mburucuyá (Mburucuyá).

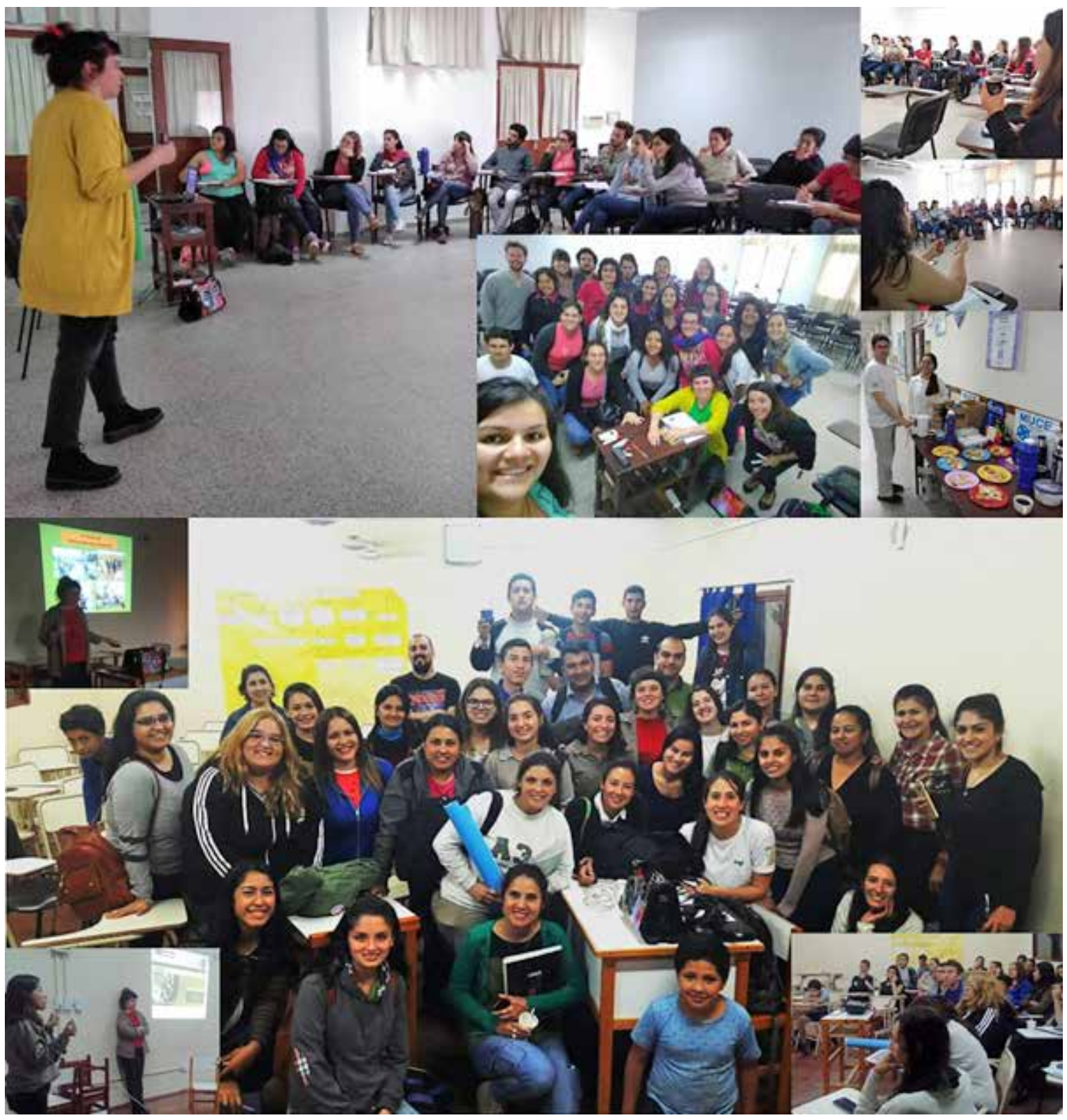

\section{Resultados y conclusiones}

Los encuentros y los talleres han capacitado a 62 personas las cuales generaron grupalmente la elaboración de 12 proyectos tomando como iniciativa posible soluciones hacia problemáticas locales de la ciudad de Corrientes y de Mburucuyá (Tabla 1). Se estima que cada participante pueda lograr transmitir sus conocimiento a, al menos, 20 personas por lo que se calcula una llegada de la información a 
Fig. 4: Espacios de intercambio y divulgación de las actividades del proyecto en Jornadas de la FaCENA (Corrientes, Argentina) y en Asunción (Paraguay).

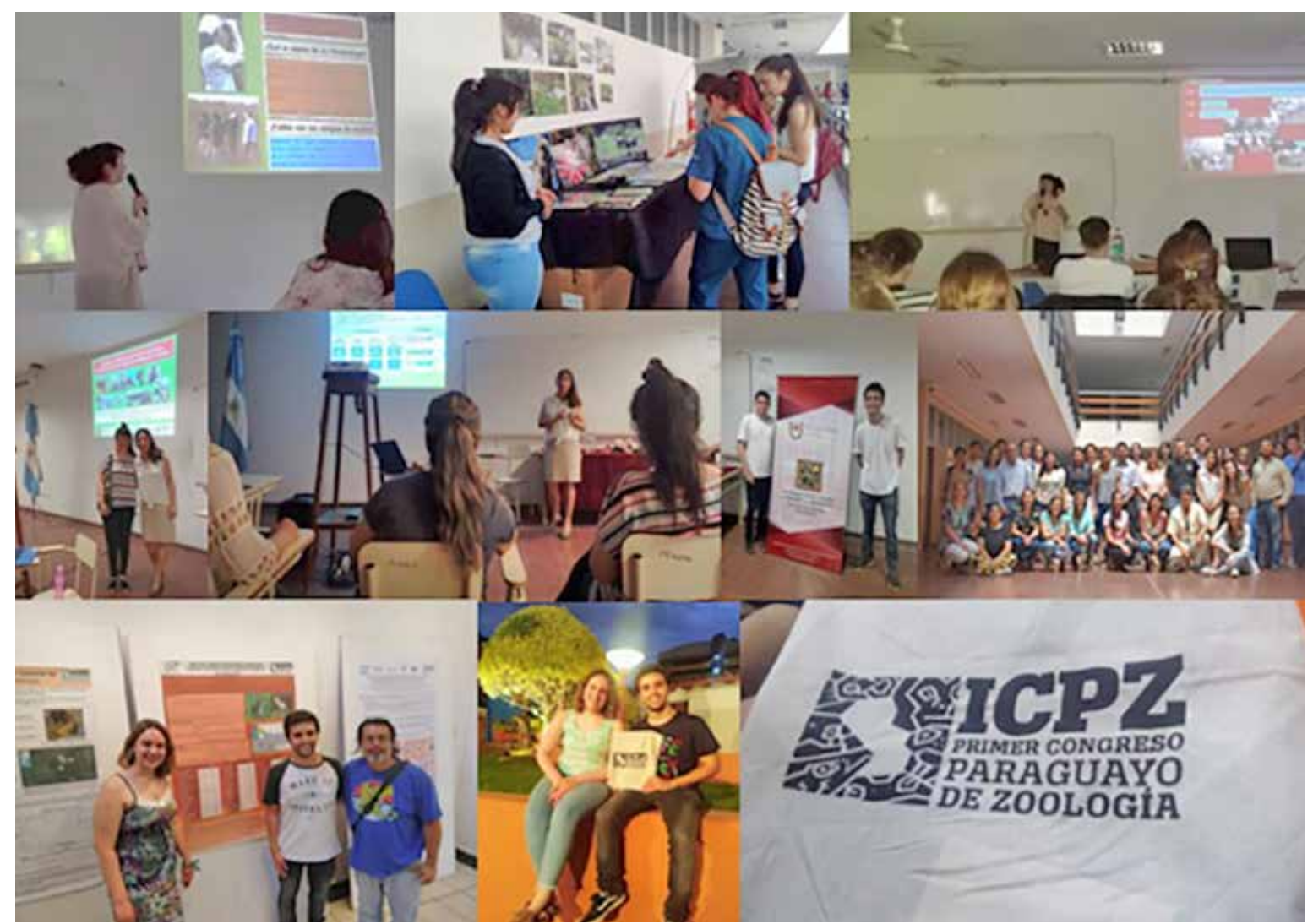

1200 personas aproximadamente. Además, este proyecto se ha vinculado no solo con las instituciones co-participantes, como la EBCo y el ISFD, también interactuó con el Parque Provincial San Cayetano y miembros de la comunidad universitaria de la Facultad de Ciencias Veterinarias, guías de turismo, naturalistas, docentes y personas interesadas en asuntos de Salud ambiental y conservación.

Asimismo, se participó activamente en espacios de divulgación digital como el diario de la FaCENA y de Rectorado, en jornadas presenciales (Fig. 4), entre ellas la XXXVIII Jornadas de Actualización en Ciencias Veterinarias los días 19 al 21 de septiembre 2019 con la exposición del Dr. Martín M. Kowalewski denominada "La conservación de los primates en Argentina: estrategias y desafíos" en Va Giardino, provincia de Córdoba. Y, el 27 de septiembre en las Jornadas Científicas y de Educación en Ciencias Biológicas de la FaCENA con una exposición de la Dra Mariana Raño, ciudad de Corrientes. El 25 y 29 de noviembre, en el Primer encuentro de Extensionismo de FaCENA (UNNE) y Jornadas de Extensionismo de UNNE (Fac. 
Cs. Económicas) se presentaron los trabajos efectuados con el proyecto de UNNE+SALUD, a cargo de la Dra. Verónica L. Romero. Y una presentación en el Primer Congreso de Zoología en Asunción Paraguay el día 29 de noviembre de 2019, a cargo de una de Débora Gilles como futura parabiologa.

Por lo tanto, se lograron concretar los objetivos propuestos y las bases para el establecimiento de la red de parabiólogos y parabiólogas en la provincia de Corrientes, que a futuro promoverán espacios de intercambio y una fuente en el ejercicio de la Ciencia ciudadana y el empoderamiento de las comunidades hacia la búsqueda de posibles soluciones de sus problemáticas ambientales locales, siendo parte del ejercicio en la toma de decisiones en la gestión de la Salud de los ecosistemas que habitan.

Tabla 1: Lista de Proyectos presentados por los participantes y evaluados por el equipo de trabajo. Resultado del cuarto encuentro noviembre y diciembre 2019. ¿Nos sería bueno poner una línea sobre cada proyecto? ¿O palabras clave? Para dar una idea de las problemáticas que abordaron

\begin{tabular}{|c|c|}
\hline Lugar & Nombre del Proyecto \\
\hline $\begin{array}{l}\text { Ciudad de Corrientes, } \\
\text { Corrientes }\end{array}$ & $\begin{array}{l}\text { "No se protege lo que no se conoce" } \\
\text { "Concientización para mejorar el cuidado de la Biodiver- } \\
\text { sidad" } \\
\text { "Conociendo los aulladores del Campus Sgto. Cabral" } \\
\text { "Mono carayá: especie centinela en la Fiebre Amarilla y } \\
\text { su prevención" } \\
\text { "Carayás en acción" }\end{array}$ \\
\hline $\begin{array}{l}\text { Mburucuyá, } \\
\text { Mburucuyá }\end{array}$ & $\begin{array}{l}\text { "Papel-yerb" } \\
\text { "Ñañangareko ñanderóga" } \\
\text { "Simbiosis" } \\
\text { "Los Monos Carayá a orillas del pueblo de Mburucuyá, } \\
\text { Corrientes" } \\
\text { "Esa onda no va" } \\
\text { "Los zorros de Mburucuyá" } \\
\text { "Plantando conciencia" }\end{array}$ \\
\hline
\end{tabular}




\section{Bibliografía}

BONILLA-ALDANA D. K., K. DHAMA and A. J. RODRIGUEZ-MORALES. 2020. Revisiting the One Health Approach in the Context of COVID-19: A look into the Ecology of this Emerging Disease. Adv. Anim. Vet. Sci., 8(3): 234-237.

CUELLAR E. y A. J. NOSS. 2014. Diversidad de mamíferos y participación local en la conservación en el Gran Chaco Boliviano. Therya5 (1): 39-60.

DUBOISSET-BROUST, L. 2013. Creating partnerships between governmental authorities and Indigenous peoples: a new strategy for biodiversity conservation. Master Thesis, Department of Earth Sciences, Uppsala University, Uppsala.

GILLESPIE T. R., C. L. NUNN and F. H. LEENDERTZ. 2008. Integrativeapproaches to the study of primate infectious disease:implications for biodiversity conservation and global health.Yearbook of Physical Anthropology 51:53-69.

HALLIDAY, JO E. B., A. L. MEREDITH, D. L. KNOBEL, D. J. SHAW, B. M. D. C. BRONSVOORT and S. CLEAVELAND. 2007. A Framework for Evaluating Animals as Sentinels for Infectious Disease Surveillance, Journal of the Royal Society, Interface / the Royal Society 4 (16): 973-84.

JONES K. E., N. G. PATEL, M. A. LEVY, A. STOREYGARD, D. BALK, J. L. GITTLEMAN and P. DASZAK. 2008. Global trends in emerging infectious diseases, Nature, 451, 990-993.

KOWALEWSKI, M. M. et al. 2011. Black and Gold Howler Monkeys (Alouattacaraya) as Sentinels of EcosystemHealth: Patterns of Zoonotic Protozoa Infection Relative to Degreeof Human-Primate Contact. American Journal of Primatology 73:75-83.

KOWALEWSKI, M. M. and T. R. GILLESPIE. 2018.Disturbance-tolerantprimates as sentinelsfor global healthand biodiversityconservation. En: Primatology, Biocultural Diversity and Sustainable Development in Tropical Forests. UNESCO Mexico, 270 - 281.

NATALINI, B. 2018. "Dinámica de enfermedades y el uso de especies centinelas para la conservación de la biodiversidad”. JORNADAS DE FAUNA SILVESTRE, Facultad de Ciencias Veterinarias, Universidad Nacional del Nordeste, Corrientes.

NATALINI, B., R. JULIÁ BAY, V. ROMERO, M. RAÑO, S. GENNUSO and M. KOWALEWSKI. Perfiles endo parasitológicos de canidos silvestres y domésticos del Parque Nacional Mburucuyá y su área de interfaz, Simposio: Desafíos en 
la interfaz doméstico-silvestre: estudios parasitológicos en mamíferos y aves de Argentina, VIII CONGRESO ARGENTINO DE PARASITOLOGÍA, 24, 25, 26 y 27 de abril de 2019, Corrientes, Argentina.

NAVARRO GÓMEZ, A. y A. RUIZ SALGADO. 2016. Importancia Social del Medio Ambiente y la Biodiversidad. Asociación de fundaciones para la conservación de la Naturaleza y Fundación Biodiversidad, Ministerios de Agricultura, Alimentación y Medio Ambiente, Burgos.

REIFJ. S. 2011. Animal Sentinels for Environmental and Public Health. Public Health Reports, supplement 1 (126): 50-57.

RODRIGUEZ MORALES, V. et al. 2011. La protección del medio ambiente y la salud, un desafío social y ético actual. Revista Cubana de Salud Pública 37 (4): 510-518.

SCHMELLER, D. S., F. COURCHAMP and G. KILLEEN. Biodiversity loss, emerging pathogens and human health risks. Biodivers. Conserv, 29: 3095-3102.

WOOLHOUSE M. E., D. T. HAYDON and R. ANTIA. 2005. Emerging pathogens: the epidemiology and evolution of species jumps. Trends Ecol. Evol., 20: 238244. 